\title{
Two-Stage Magnetization Reversal in Exchange Biased Bilayers
}

\author{
C. Leighton, ${ }^{1}{ }^{*}$ M. R. Fitzsimmons, ${ }^{2}$ P. Yashar, ${ }^{2}$ A. Hoffmann, ${ }^{2}$ J. Nogués, ${ }^{3}$ \\ J. Dura, ${ }^{4}$ C. F. Majkrzak, ${ }^{4}$ and Ivan K. Schuller ${ }^{1}$ \\ ${ }^{1}$ Physics Department, University of California-San Diego, La Jolla, California 92093-0319 \\ ${ }^{2}$ Los Alamos National Laboratory, Los Alamos, New Mexico 87545 \\ ${ }^{3}$ Departament de Física, Universitat Autònoma de Barcelona, 08193 Bellaterra, Spain \\ ${ }^{4}$ National Institute of Standards and Technology, Gaithersburg, Maryland 20899
}

(Received 2 August 2000)

\begin{abstract}
$\mathrm{MnF}_{2} / \mathrm{Fe}$ bilayers exhibit asymmetric magnetization reversal that occurs by coherent rotation on one side of the loop and by nucleation and propagation of domain walls on the other side of the loop. Here, we show by polarized neutron reflectometry, magnetization, and magnetotransport measurements that for samples with good crystalline "quality" the rotation is a two-stage process, due to coherent rotation to a stable state perpendicular to the cooling field direction. The result is remarkably asymmetrically shaped hysteresis loops.
\end{abstract}

DOI: $10.1103 /$ PhysRevLett.86.4394

Despite recently renewed interest in the phenomenon of exchange anisotropy [1] at the interface between antiferromagnets $(\mathrm{AF})$ and ferromagnets $(\mathrm{F})$, a full understanding is elusive [2]. A complete description of exchange anisotropy would incorporate an explanation of the mechanism for exchange biasing [2-8], the effects of interface disorder [9-11], the relationship between the exchange bias $\left(H_{E}\right)$ and the coercivity $\left(H_{C}\right)[6,12,13]$, as well as an understanding of the magnetization reversal mechanisms [14-16]. In an attempt to address these issues, we have focused on a model thin film system $\mathrm{TMF}_{2} / \mathrm{Fe}(\mathrm{TM}=$ transition metal), with which we have been able to elucidate perpendicular coupling [17], effects of compensation [18] and interfacial disorder [9,10], and the relationship between $H_{E}$ and $H_{C}$ [12].

A recent thrust has been to understand the mechanisms by which the magnetization reverses in such systems [15]. This is a fundamental point, clearly related to the behavior of $H_{C}$ (zero moment half-width of the hysteresis loop), which is considered important [6]. Although there are few more fundamental questions about the nature of a hysteresis loop than the issue of the magnetization reversal mechanism, it has largely escaped investigation in exchange biased systems. Recent papers $[14,15]$ have contributed to the realization that the magnetization reversal is intrinsically asymmetric under certain conditions. This naturally explains the often observed [1,18-20] and discussed [16] asymmetrically shaped loops, which have evaded explanation for over 40 years. Moreover, it appears that this is a general phenomenon after observation in $\mathrm{FeMn} / \mathrm{Py}$ (Permalloy) [14], $\mathrm{MnF}_{2} / \mathrm{Fe}, \mathrm{FeF}_{2} / \mathrm{Fe}$ [15], $\mathrm{NiO} / \mathrm{Co}$ [21], and even spin-valve-type materials [22]. In summary, it is clear that reversal asymmetry in exchange biased systems is a general phenomenon requiring immediate attention.

In the $\mathrm{MnF}_{2} / \mathrm{Fe}$ and $\mathrm{FeF}_{2} / \mathrm{Fe}$ systems, we have shown that for certain cooling field orientations the magnetization reverses by coherent rotation on the high field side of the loop (i.e., where the coercive field is $\left|H_{E}\right|+H_{C}$ ) and by
PACS numbers: $75.70 . \mathrm{Cn}, 75.30 . \mathrm{Gw}$

domain wall nucleation and propagation on the low field side of the loop (i.e., where the coercive field is $H_{C}-$ $\left.\left|H_{E}\right|\right)$, a very clear asymmetry [15]. The magnetization hysteresis loops show very slight shape asymmetry, which must be associated with this effect [23]. The asymmetry is easily understood on consideration of the twinned nature of the AF films (Fig. 1). When the cooling field bisects the twin anisotropy axes (as shown), the frustration of the coupling between the individual AF twins and the ferromagnetic overlayer leads to an effective " $45^{\circ}$ coupling," resulting in the easy axes shown in the figure. Note that the unidirectional anisotropy (UA) axis provides a global energy minimum, while the other three axes represent local easy axes. When the field is reduced from positive saturation $\left(H \| H_{F C}\right)$, the magnetization rotates to the easy axis at $90^{\circ}$ and eventually is fully reversed. At this point, as the field is reduced from negative saturation, the unidirectional anisotropy stimulates nucleation of domains with

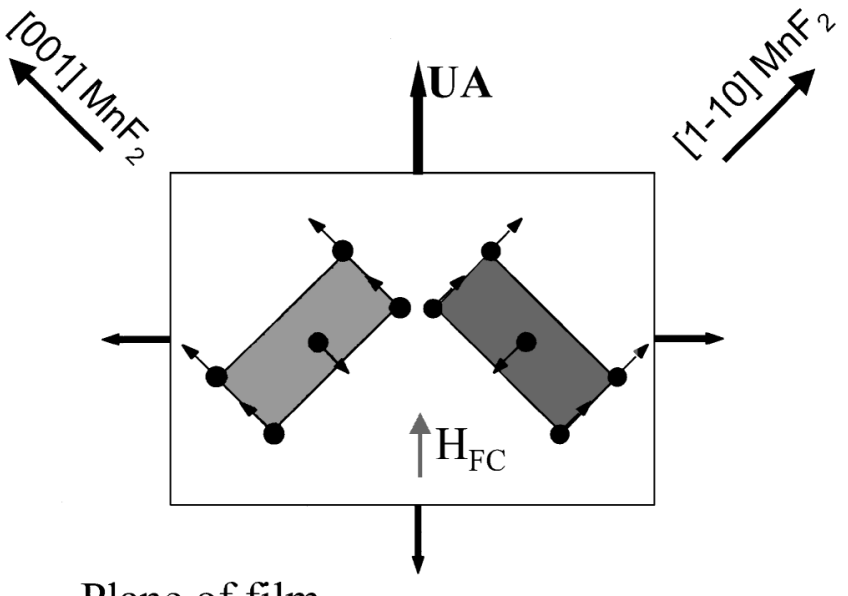

Plane of film

FIG. 1. Schematic showing the two $\mathrm{MnF}_{2}$ twins, the unidirectional anisotropy direction (UA) and the cooling field $\left(H_{F C}\right)$ orientation. Solid arrows represent easy axes. 
magnetization parallel to $H_{F C}$ and rotation is suppressed, i.e., the reversal processes are different.

Here, we use the complementary techniques of magnetometry, polarized neutron reflectometry (PNR), and anisotropic magnetoresistance (AMR) to show that the rotation can occur in two stages where the magnetization vector falls into a potential minimum at $90^{\circ}$ to $H_{F C}$. This leads to a remarkably asymmetric "kinked" hysteresis loop never previously observed. The requirement to observe this is a low mosaicity measured by the width of the $\mathrm{x}$-ray diffraction rocking curve through $\mathrm{MnF}_{2}$ reflections.

Details of the sample preparation and characterization were given in previous publications [10,12]. The layer structure is $\mathrm{MgO}(100) / \mathrm{ZnF}_{2}(110) / \mathrm{MnF}_{2}(110) / \mathrm{Fe} / \mathrm{Al}$ (cap layer), with thicknesses 250/500/120/50 ̊, respectively. High angle x-ray diffraction (with the scattering vector both in the sample plane and perpendicular to it), grazing incidence reflectivity, and reflection high energy electron diffraction revealed twinned AF layers, polycrystalline $\mathrm{Fe}$ overlayers, and a controllable interface roughness [10]. The twinning is critical to the understanding of the exchange biasing. An important parameter when assessing structural data is the full width at half maximum (FWHM) of the transverse scan through the $\mathrm{MnF}_{2}(110)$ reflection, $\Delta \theta$. This is extremely sensitive to the substrate temperature during deposition, even more so than the roughness. We find that observation of the "kink" feature discussed below is strongly correlated with low values of $\Delta \theta$.

The measurements consist of SQUID magnetometry and AMR from 100 to $4.2 \mathrm{~K}$ in fields up to $70 \mathrm{kOe}$, and PNR [24] at $36 \mathrm{~K}$ in low magnetic fields. The PNR measurements were made using the NG1 reflectometer at the National Institute for Standards and Technology. Briefly, a polarized neutron beam is specularly reflected by the sample onto a polarization analyzer and detector. Two nonspin-flip cross sections (i.e., polarizations unchanged by the sample) were measured in addition to the spin-flip scattering intensity. The spin-flip scattering intensity arises from a component of the sample magnetization vector perpendicular to the applied field which flips the neutron polarization from up to down or vice versa. The measurements presented here were made by saturating the film in one direction then increasing the field in the opposite direction to the coercive point.

A magnetization hysteresis loop for a sample with $\Delta \theta=$ $1.9^{\circ}$ is shown in Fig. 2 at $10 \mathrm{~K}$ after field cooling in a 234 Oe field applied as in Fig. 1. The asymmetric shape, with a distinct kink on the left side of the loop, is striking. The width of this kink is a strong function of $\Delta \theta$, as shown in the inset of Fig. 2. Clearly, only the samples with the lowest FWHM of the high angle $\mathrm{MnF}_{2} \mathrm{x}$-ray rocking curves display this effect. Note that $\Delta H$ is uncorrelated with other structural parameters such as interface roughness and correlation length. Another important point is that the kink occurs very close to $m=0$, where $m$ is the magnitude of the sample magnetic moment projected onto

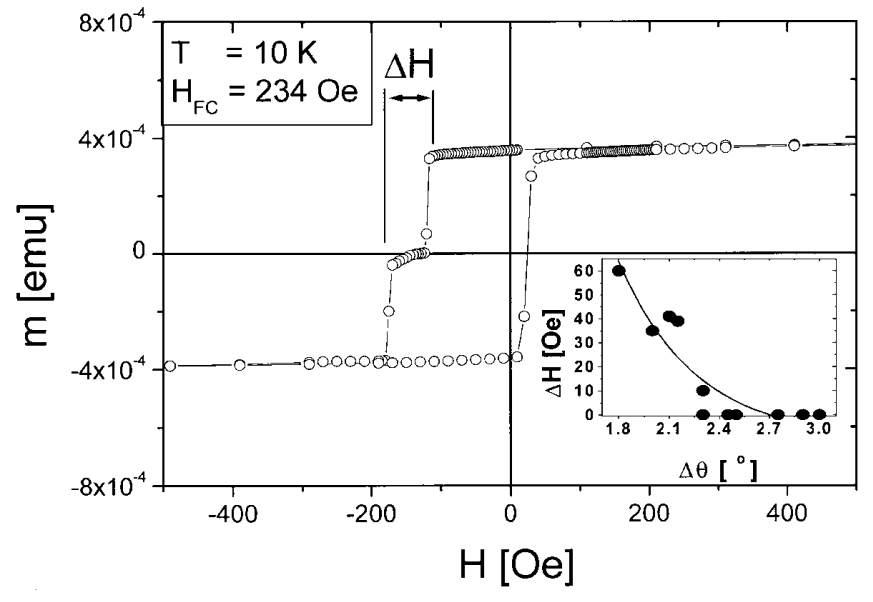

FIG. 2. A hysteresis loop at $T=10 \mathrm{~K}$ and $H_{F C}=234$ Oe. Inset: $\Delta H$ vs $\Delta \theta$. Solid line is a guide to the eye.

the field direction. Since the magnetization reversal on the high field side of the loop is accomplished by rotation (as observed with PNR), and given that an easy axis is present at $90^{\circ}$ to the field cooling direction, it is natural to propose that this kink feature is due to rotation to this easy axis. In other words, the magnetization reversal occurs in two distinct stages: first, by coherent rotation through $90^{\circ}$ at which point the system falls into a stable configuration, followed by further rotation to $180^{\circ}$ after increasing the field by $\Delta H$. Hence, the "shelflike" feature near $m=0$ corresponds to the range of fields for which the magnetization vector is in a metastable state at $90^{\circ}$ to the original magnetization direction. The absence of the kink on the right-hand side of the loop is due to the fact that reversal here occurs by domain wall nucleation and propagation.

An important observation is that the minor loops are reversible on the high field side; i.e., if we sweep from positive saturation to the kink and then reverse the field and move back up the loop, we observe fully reversible behavior. This is in contrast to the other side of the loop where clear irreversibility is evident. This must be seen as strong evidence for our model: The coherent rotation process is reversible while the domain processes are not. In particular, this rules out any possibility of a metastable domain state which is achieved near $m=0$ on the high field side.

The $H_{F C}$ dependence of this effect is shown in Fig. 3, with the usual positive bias at high cooling fields $[10,12]$. The three hysteresis loops in this figure correspond to low $H_{F C}(234 \mathrm{Oe})$ where $H_{E}<0$, intermediate $H_{F C}(10 \mathrm{kOe})$ where $H_{E}=0$, and high $H_{F C}(70 \mathrm{kOe})$ where $H_{E}>0$. The kink, which originally occurs on the left side of the loop at low $H_{F C}$, disappears when the net $H_{E}$ vanishes and occurs on the right side of the loop for positive $H_{E}$. The appearance of the anomaly on the right side of the loop for $H_{E}>0$ is expected as this is the side of the loop for which we believe coherent rotation now takes place (i.e., the high field side [15]), while domain wall processes account for the reversal on the other side. The absence of a kink feature for a sample cooled such that $H_{E}=0$ is consistent with 

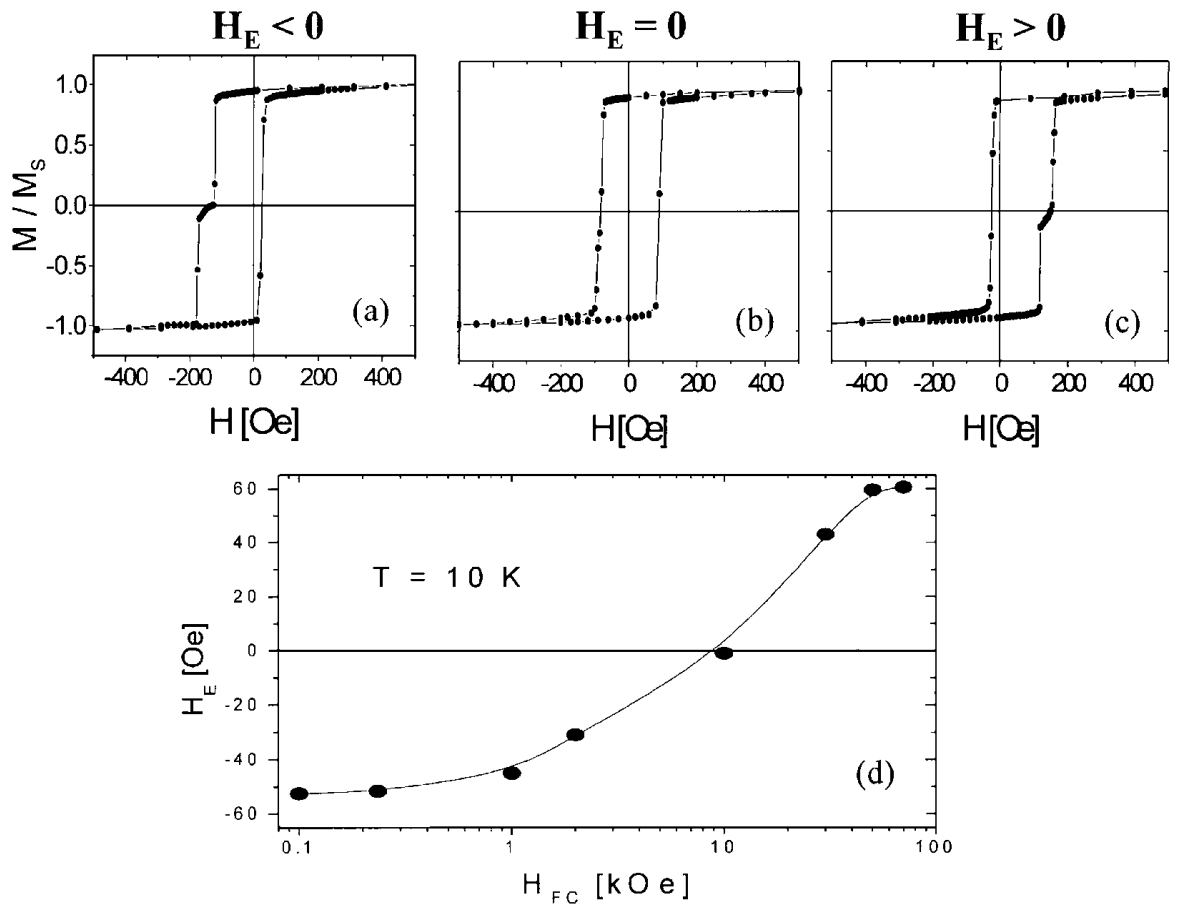

FIG. 3. The $H_{F C}$ dependence of $H_{E}$ and the kink feature. (d) shows the $H_{F C}$ dependence of $H_{E}$. The solid line is a guide to the eye. The upper three figures are loops taken at fields such that $H_{E}$ is negative (a), zero (b), and positive (c), respectively.

PNR observation of domain wall processes on both sides of the loop. In essence, the kink always occurs on the high field side of the loop, disappearing when the left and right sides cross $m=0$ at the same field magnitude, i.e., when $H_{E}\left(H_{F C}\right)=0$.

The kink width $\Delta H$ is further examined in Fig. 4 where $\Delta H$ and $H_{E}$ are plotted against $T$ after the same field cooling procedure. The identical temperature dependencies of $H_{E}$ and $\Delta H$ are further evidence that the kink is intimately related to the unidirectional anisotropy. This temperature dependence has been discussed previously [10] and has been investigated as a function of interface disorder [25].

As discussed in a previous publication [23], the reversal asymmetry in this system can also be probed by AMR. The inset of Fig. 4 shows AMR at $10 \mathrm{~K}$ under identical conditions to those of Fig. 2, with the sample current, I $\| \mathbf{H}$. The sensitivity of the resistance to the angle between $\mathbf{M}$ and I usually results in a symmetric AMR response with two minima of identical magnitude at $\pm H_{C}$. Here, the striking asymmetry in the sizes of the magnetoresistive response on the left- and right-hand sides of the loop is again due to the rotation on the left side (where a large AMR response is observed) and domain wall nucleation and propagation on the right (where the AMR response is a factor of 6 smaller). The asymmetry disappears at $T>T_{N}$ [26] and does not exist at all in $\mathrm{ZnF}_{2} / \mathrm{Fe}$ bilayers with comparable high angle rocking curve widths. Close inspection of the AMR on the coherent rotation side of the loop reveals a "flat-bottomed" shape, as shown in Fig. 5(a), which correlates with the kink in the magnetization hysteresis loop. This is the field region where the magnetization vector is in a potential minimum at $90^{\circ}$ to $H_{F C}$, and the vector mag- netization is constant; i.e., the angle between $\mathbf{M}$ and $\mathbf{I}$, is not varying in this region. Absolute proof of this hypothesis is shown in Fig. 5(b) where the intensity of the neutron spin-flip scattering cross section, $I_{S F}$, is plotted in the same figure as the AMR response. This scattering is due to the existence of some component of the film magnetization perpendicular to $H_{F C}$. Hence, the region of constant $I_{S F}$ corresponds to a static net magnetization vector. The spin-flip scatter is observed to be strong over the whole high field side of the loop, and essentially zero on the low field side of the loops.

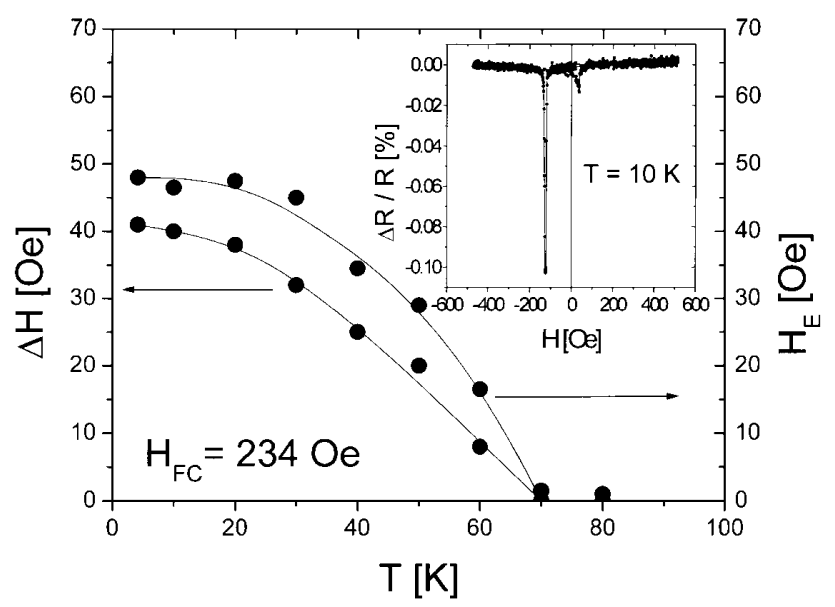

FIG. 4. The $T$ dependencies of $H_{E}$ and $\Delta H$ at $H_{F C}=234$ Oe. Note that to determine $H_{E}$ we explicitly assume that the coercive fields on either side of the loop are identical; i.e., we define $H_{E}$ as the field displacement of the loop center. Inset: AMR at $T=10 \mathrm{~K}$ and $H_{F C}=234$ Oe. Note: $\Delta R / R_{0}=\left(R-R_{0}\right) / R_{0}$, where $R_{0}$ is the resistance at 500 Oe. 

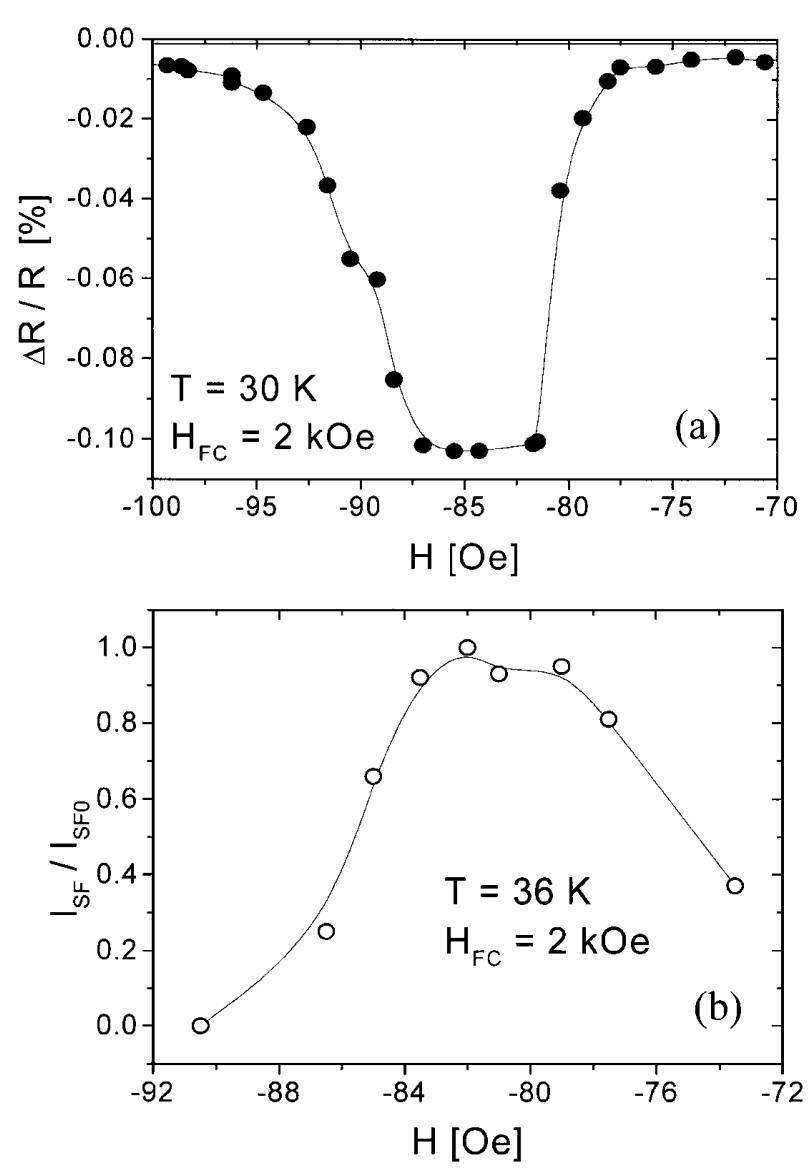

FIG. 5. "Close-up" of the AMR (a) and normalized neutron spin-flip scattering intensity (b) near the kink feature. $I_{S F}$ is normalized to the maximum intensity $I_{S F 0}$. Note that the slight field discrepancy between the two curves is due to the difficulty in accurately determining the field during the neutron scattering measurements, and a slightly different measuring temperature. Note also the slight discrepancy in cooling field values compared to Fig. 2.

In summary, we have provided conclusive evidence that the magnetization reversal by coherent rotation in $\mathrm{MnF}_{2} / \mathrm{Fe}$ bilayers can occur via a two-stage process. This process produces a strikingly asymmetric, kinked hysteresis loop which can be naturally explained by the previously inferred magnetic anisotropy [27]. Clearly, asymmetry in the magnetization reversal process in exchange biased systems is of great importance in terms of dictating the nature of the hysteresis and should be considered in thorough investigation of new systems.

We thank K. Liu, B. J. Jönsson-Åkerman, M. Song, T. C. Schulthess, M. Pardavi-Horvath, and NIST (for the use of neutron facilities). This work was supported by the U.S. DOE BES-DMS, Contract No. W-7405-Eng-36, Grant No. DE-FG03-87ER-45332 (LANL), U.S. DOE (UCSD), UC-CULAR and Catalan DGR (1999SGR00340) (J. N.).

*Present address: Department of Chemical Engineering and Materials Science, University of Minnesota, Minneapolis, MN 55455.
[1] W.H. Meiklejohn and C.P. Bean, Phys. Rev. 105, 904 (1957).

[2] See the review, J. Nogués and I. K. Schuller, J. Magn. Magn. Mater. 192, 203 (1998).

[3] D. Mauri, H. C. Siegmann, P. S. Bagus, and E. Kay, J. Appl. Phys. 62, 3047 (1987).

[4] A. P. Malozemoff, J. Appl. Phys. 63, 3874 (1988).

[5] N. C. Koon, Phys. Rev. Lett. 78, 4865 (1997).

[6] T. C. Schulthess and W. H. Butler, Phys. Rev. Lett. 81, 4516 (1998); J. Appl. Phys. 85, 5510 (1999).

[7] M. Kiwi, J. Mejía-Lopez, R. D. Portugal, and R. Ramirez, Europhys. Lett. 48, 573 (1999); Appl. Phys. Lett. 75, 3995 (1999).

[8] K. Takano, R. H. Kodama, A. E. Berkowitz, and W. Cao, Phys. Rev. Lett. 79, 1130 (1997).

[9] J. Nogués, D. Lederman, T. J. Moran, I. K. Schuller, and K. V. Rao, Appl. Phys. Lett. 68, 3186 (1996); J. Nogués, D. Lederman, T. J. Moran, and I. K. Schuller, Phys. Rev. Lett. 76, 4624 (1996).

[10] C. Leighton, J. Nogués, H. Suhl, and I. K. Schuller, Phys. Rev. B 60, 12837 (1999).

[11] C.-M. Park, K.-I. Min, and K. H. Shin, J. Appl. Phys. 79, 6228 (1996).

[12] C. Leighton, J. Nogués, B. J. Jonsson-Åkerman, and I. K. Schuller, Phys. Rev. Lett. 84, 3466 (2000).

[13] D. V. Dimitrov, S. Zhang, J. Q. Xiao, G. C. Hadjipanayis, and C. Prados, Phys. Rev. B 58, 12090 (1998).

[14] V. I. Nikitenko, V. S. Gornakov, A. J. Shapiro, R. D. Shull, K. Liu, S. M. Zhou, and C. L. Chien, Phys. Rev. Lett. 84, 765 (2000).

[15] M. R. Fitzsimmons, P. C. Yashar, C. Leighton, J. Nogués, J. Dura, C. F. Majkrzak, and I. K. Schuller, Phys. Rev. Lett. 84, 3986 (2000).

[16] M.D. Stiles and R. McMichael, Phys. Rev. B 59, 3722 (1999).

[17] T. J. Moran, J. Nogués, D. Lederman, and I. K. Schuller, Appl. Phys. Lett. 72, 617 (1998).

[18] J. Nogués, T. J. Moran, D. Lederman, I. K. Schuller, and K. V. Rao, Phys. Rev. B 59, 6984 (1999).

[19] C. Tsang and K. Lee, J. Appl. Phys. 53, 2605 (1982).

[20] T. Ambrose and C.L. Chien, J. Appl. Phys. 83, 7223 (1998).

[21] H. D. Chopra, D. X. Yang, P. J. Chen, H. J. Brown, L. J. Swartzendruber, and W. F. Egelhoff, Jr., Phys. Rev. B 61, 15312 (2000).

[22] X. Portier, A. K. Petford-Long, A. de Morais, N.W. Owen, H. Laidler, and K. O'Grady, J. Appl. Phys. 87, 6412 (2000).

[23] C. Leighton, M. Song, J. Nogués, M. C. Cyrille, and I. K. Schuller, J. Appl. Phys. 88, 344 (2000).

[24] C. F. Majkrzak, Physica (Amsterdam) 221B, 342 (1996).

[25] M. R. Fitzsimmons, C. Leighton, A. Hoffmann, P.C. Yashar, J. Nogués, I. K. Schuller, C. F. Majkrzak, J. A. Dura, and H. Fritzsche (unpublished).

[26] The $T$ dependence of the ratio of the AMR responses on either side of the loop is similar to that of the "asymmetry field" in Ref. [25].

[27] Note: We recently became aware of an independent theoretical study [Z. Li and S. Zhang, Appl. Phys. Lett. 77, 423 (2000)] of reversal processes in exchange biased systems which finds discontinuous jumps in magnetization. 